\title{
Análisis comparativo de suelos de cinco distritos de la cuenca Chinchipe y su influencia en la fertilidad del cultivo de café (Coffea arábica l.)
}

\section{Comparative analysis of soils in five districts of the Chinchipe basin and their influence on the fertility of coffee cultivation (Coffea arabica 1.)}

\author{
Noly C Vílchez Parra ${ }^{1}$, Jeimis R Yalta $\mathrm{M}^{2}$ y Victor B Carril F
}

\begin{abstract}
RESUMEN
En la investigación se determinó el análisis comparativo de suelos de cinco distritos pertenecientes a la cuenca Chinchipe, ubicada en la Provincia de San Ignacio, que producen café (Coffea arábica) de especialidad. Las muestras de suelos, fueron colectadas teniendo en cuenta, textura, color, topografía y otras características, donde cada una de ellas estuvo constituida por 20 submuestras, con un peso aproximado de $1.0 \mathrm{~kg}$, tomadas a una profundidad de $30 \mathrm{~cm}$; luego fueron secadas al aire. Se obtuvieron siete análisis de suelo, que fueron enviados al Laboratorio de Química Agrícola del Instituto Rural Valle Grande. Los resultados nos arrojan que, los suelos presentan en promedio, una clase textural que corresponde al tipo Franco; $\mathrm{pH}$ de 5.16; materia orgánica en 4.40 $\%$; porcentaje de acidez intercambiable de $26.43 \%$; y una capacidad de intercambio catiónico efectivo de 16.46 $\mathrm{mEq} / 100 \mathrm{~g}$. Estas características, nos permiten concluir que se trata de suelos con alta fertilidad, apropiados para el cultivo, y hacen deseables para conseguir resultados satisfactorios para el café de especialidad o de alta calidad organoléptica.
\end{abstract}

Palabras clave: café, análisis de suelos, parámetros, cuenca Chinchipe.

\begin{abstract}
In this research was determined the comparative analysis of soils from five districts belonging to the Chinchipe basin. These districts are located in the province of San Ignacio which produces specialty coffee (Coffea arabica). Soil samples were collected taking into account their texture, color, topography and other characteristics. Each sample consisted of 20 subsamples, weighing approximately $1.0 \mathrm{~kg}$. These samples were taken at a depth of $30 \mathrm{~cm}$ and then they were air dried. We obtained seven soil analyzes, which were sent to the Agricultural Chemistry Laboratory of the Valle Grande Rural Institute. The results were processed and the soils have a textural class that corresponds to the Franco type; $\mathrm{pH}$ of 5.16; organic matter of $4.40 \%$; interchangeable acidity percentage of $26.43 \%$; and one of effective cation exchange capacity of $16.46 \mathrm{mEq} / 100 \mathrm{~g}$. These characteristics allow us to conclude that these are soils with high fertility, suitable for cultivation, and make them desirable to achieve satisfactory results for specialty coffee or of high organoleptic quality.
\end{abstract}

Keywords: coffee, soil analysis, parameters, Chinchipe basin.

1. Universidad Nacional de Jaén, Perú. Email: ncvip@ hotmail.com

2. Universidad Nacional de Jaén, Perú. Email: jryalta@gmail.com

3. Universidad Nacional de Jaén, Perú. Email: vicben20@gmail.com 


\section{INTRODUCCIÓN}

Los aspectos físicos y químicos de los suelos permiten realizar diagnósticos de la textura y estructura del suelo. El café produce cosechas adecuadas en suelos desarrollados en diferentes formaciones geológicas y bajo condiciones climáticas variables. La textura y su profundidad son de vital importancia. En suelos compactos o poco profundos, las raíces no se extienden más que en los horizontes superficiales, acarreando problemas en la productividad.

Los suelos adecuados para el café, deben ser del tipo franco, drenados, rico en macro y micro nutrientes, particularmente en potasio y boro y con alto contenido de materia orgánica. Por lo general, el pH apropiado para el café debe estar en el rango de 4.5 a 6.0. Con respecto a la fertilidad, es muy importante la capacidad de intercambio de cationes (CICE), parámetro que indica si un suelo posee baja o alta fertilidad, siendo crucial en plantaciones con grandes rendimientos, en donde, las deficiencias nutricionales son visibles en un corto tiempo.

El café es un cultivo que responde bien a la fertilización balanceada, por lo tanto, altos rendimientos sostenibles, solo son posibles si se conoce la extracción continua de elementos por cosecha en el año, en un mismo suelo; siendo entonces el análisis de suelo, una herramienta importante, para realizar el diagnóstico del estado nutricional y tomar decisiones a posteriori.

En la cuenca Chinchipe se observa migraciones forzadas por la pobreza extrema; la agricultura migratoria y de subsistencia; las formas de uso actual de la tierra; la deforestación; el uso del fuego, han generado impactos negativos, como erosión de laderas, desaparición de la fauna silvestre, disminución de quebradas. Sin embargo, la agroforestería y, los cafés especiales como propuestas de sostenibilidad, vienen mejorando la calidad de vida de los productores de café, de los distritos en estudio (Vílchez et al., 2017).

Uno de los objetivos de los programas de extensión agrícola, que promueven los Organismos No Gubernamentales (ONG), el Ministerio de Agricultura, y Empresas Privadas que realizan operaciones de comercialización y manejo técnico del café; es el manejo y sostenibilidad de los recursos naturales de la cuenca, cuyo objetivo es reducir la presión sobre los recursos, siendo la agricultura no tecnificada y migratoria una de las principales amenazas (Arcos et al., 2007).

El presente trabajo de investigación, pretende evidenciar que el cultivo del café, en esta parte de la cuenca, puede mejorar su rendimiento y productividad, si se tienen políticas, estrategias y herramientas que ayuden a gestionar su calidad en taza y rendimiento físico. Asimismo, se justifica por el impacto económico que ayudaría a los productores a realizar fertilizaciones adecuadas o balanceadas a un mínimo costo. Además, pretende repotenciar los tres ejes del desarrollo sostenible, 
los mismos que son: Económico, Social y Ecológico para mejorar la calidad de vida de la población de esta parte de la cuenca del Chinchipe. Por ello, la investigación, tuvo como objetivo general el análisis comparativo de cinco parámetros de suelos de la cuenca Chinchipe y su influencia en la fertilidad del cultivo de café (Coffea arábica L.).

\section{MATERIALES Y MÉTODOS}

\section{Localización.}

La fase de campo se llevó a cabo en cinco distritos (San Ignacio, San José de Lourdes, Chirinos, Huarango y Namballe), ubicados dentro de la cuenca del Chinchipe en la Provincia de San Ignacio, siendo sus coordenadas geográficas 59'0” Latitud Sur y 790'0” Longitud Oeste, UTM 721702, donde se presentan las siguientes condiciones medias: Temperatura $21,7^{\circ} \mathrm{C}$, humedad relativa mayor a $70 \%$, precipitación total anual $1165 \mathrm{~mm}$. La temperatura promedio anual no constituye una pauta definitiva para señalar si un área es apropiada para establecer cafetales (Rimache, 2008). Las parcelas de café donde se llevaron a cabo el muestreo de suelos, estuvieron compuestas por las variedades Typica, Caturra, Catimor y Pache, sembrados en sistema agroforestal, con distanciamiento promedio entre $1.20 \mathrm{~m}$ entre plantas y por $1.80 \mathrm{~m}$ entre líneas.

\section{Metodología.}

El componente suelo se ha evaluado a través de registros o muestras de suelos tomadas en las parcelas de los diversos sectores de la cuenca Chinchipe, lográndose tener un total de siete registros de análisis de suelos. Antes de iniciarse el muestreo, se verificó in situ si las parcelas tuvieron un adecuado manejo agronómico como son, el control de malezas, manejo de plagas y, fertilización no menor a tres meses de aplicación.

Las muestras fueron colectadas entre marzo y abril de 2017, en parcelas ubicadas entre 1500 y 1800 m.s.n.m., por el equipo técnico del Programa Familia de la Empresa Comercio \& Cía. S.A., siguiendo los criterios estándar de colecta: el área de muestreo fue de 1 ha., por cada registro (7 has en total); toma de porciones de suelos a una profundidad de $30 \mathrm{~cm}$ de varios puntos de la finca (ha). Obtenida las muestras se homogenizaron, y se colocaron en bolsas, haciendo un peso aproximado de $1 \mathrm{~kg}$ por cada muestra. Estas muestras fueron rotuladas adecuadamente y, fueron llevadas al Laboratorio de Química Agrícola del Instituto Rural Valle Grande de Lima, en donde se realizó el proceso de evaluación de los parámetros (ver Tabla 1). La interpretación de los resultados de los informes de evaluación de los parámetros, se procesaron con Excel, en la Empresa Comercio \& Cia. S.A. localizada en la ciudad de Jaén. 
Análisis comparativos de suelos de cinco distritos de la cuenca Chinchipe

Tabla 1. Parámetros evaluados en los análisis de suelo de la Cuenca Chinchipe

\begin{tabular}{lccc}
\hline \multicolumn{1}{c}{ Parámetro } & Unidad & Método & Técnica \\
\hline Textura: & $\%$ & MES-001 & \\
Arena & $\%$ & MES-001 & Bouyoucos \\
Limo & $\%$ & MES-001 & \\
Arcilla & & MES-002 & Electrométrico \\
$\mathrm{pH}(1 / 1)$ a Temp. $=22{ }^{\circ} \mathrm{C}$ & $\%$ & MES-003 & Walkley y Black \\
Materia Orgánica & $\%$ & MES-004 & Cálculo Matemático \\
P.A.I. & $\mathrm{mEq} / 100 \mathrm{~g}$ & MES-005 & Cálculo Matemático \\
C.I.C.E. & & & \\
\hline
\end{tabular}

Donde:

$\%:$ Masa / Masa

(1/1): Relación masa del suelo / volumen del agua

P.A.I.: Porcentaje de acidez intercambiable. C.I.C.E.: Capacidad de intercambio catiónico efectivo.

Mes: Método propio del laboratorio.

\section{RESULTADOS}

En la Tabla 2 se muestran los resultados de los análisis de suelos realizado en las cinco zonas cafetaleras en estudio.

Tabla 2. Promedio y desviación estándar de caracteres del suelo en 5 distritos cafetaleros de la cuenca Chinchipe

\begin{tabular}{|c|c|c|c|c|c|c|c|c|c|c|c|c|c|c|c|}
\hline \multirow[b]{2}{*}{ Variable } & \multicolumn{3}{|c|}{ San Ignacio } & \multicolumn{3}{|c|}{ San José de Lourdes } & \multicolumn{3}{|c|}{ Namballe } & \multicolumn{3}{|c|}{ Huarango } & \multicolumn{3}{|c|}{ Chirinos } \\
\hline & Media & $\begin{array}{c}\text { Desv. } \\
\text { Estand. }\end{array}$ & $\begin{array}{l}\text { C.V. } \\
(\%)\end{array}$ & Media & $\begin{array}{c}\text { Desv. } \\
\text { Estand. }\end{array}$ & $\begin{array}{l}\text { C.V. } \\
(\%)\end{array}$ & Media & $\begin{array}{c}\text { Desv. } \\
\text { Estand. }\end{array}$ & $\begin{array}{l}\text { C.V. } \\
(\%)\end{array}$ & Media & $\begin{array}{c}\text { Desv. } \\
\text { Estand. }\end{array}$ & $\begin{array}{l}\text { C.V. } \\
(\%)\end{array}$ & Media & $\begin{array}{c}\text { Desv. } \\
\text { Estand. }\end{array}$ & $\begin{array}{l}\text { C.V. } \\
(\%)\end{array}$ \\
\hline Arena & & & & & & & & & & & & & & & \\
\hline$(\%)$ & 24.96 & 11.36 & $45.5 \%$ & 46.96 & 11.36 & $24.2 \%$ & 35.93 & 1.43 & $4.0 \%$ & 7.915 & 2.82 & $35.6 \%$ & 47.45 & 13.50 & $28.5 \%$ \\
\hline Limo & & & & & & & & & & & & & & & \\
\hline$(\%)$ & 45.47 & 6.41 & $14.1 \%$ & 33.97 & 14.19 & $41.8 \%$ & 49.00 & 4.24 & $8.7 \%$ & 23.45 & 6.29 & $26.8 \%$ & 35.47 & 16.31 & $46.0 \%$ \\
\hline Arcilla & & & & & & & & & & & & & & & \\
\hline$(\%)$ & 29.58 & 4.95 & $16.7 \%$ & 19.08 & 2.83 & $14.8 \%$ & 20.58 & 2.12 & $10.3 \%$ & 71.15 & 5.56 & $7.8 \%$ & 22.49 & 4.82 & $21.4 \%$ \\
\hline $\mathrm{pH}$ & 4.24 & 0.36 & $9 \%$ & 4.70 & 0.18 & $3.9 \%$ & 4.46 & 0.15 & $3.3 \%$ & 5.66 & 1.27 & $22.5 \%$ & 5.72 & 0.06 & $1.1 \%$ \\
\hline M.O. \% & 5.31 & 0.74 & $14 \%$ & 4.14 & 0.74 & $17.8 \%$ & 6.13 & 0.36 & $5.9 \%$ & 4.885 & 1.11 & $22.7 \%$ & 3.43 & 1.92 & $55.9 \%$ \\
\hline P.A.I. & 39.51 & 16.11 & $41 \%$ & 73.26 & 5.86 & $8.0 \%$ & 20.98 & 2.57 & $12.3 \%$ & 0.21 & 0.08 & $40.4 \%$ & 0.07 & 0.02 & $32.6 \%$ \\
\hline C.I.C.E. & 7.51 & 0.47 & $6 \%$ & 6.25 & 3.14 & $50.2 \%$ & 7.77 & 1.45 & $18.7 \%$ & 44.305 & 7.22 & $16.3 \%$ & 18.60 & 8.77 & $47.1 \%$ \\
\hline
\end{tabular}




\section{Textura.}

En la Figura 1 se observa los valores promedios alcanzados de las porciones arena, limo, arcillas correspondieron a 33.95\%, 33.67\%, y 32.38\% respectivamente, indicándonos la predominancia del suelo tipo Franco.

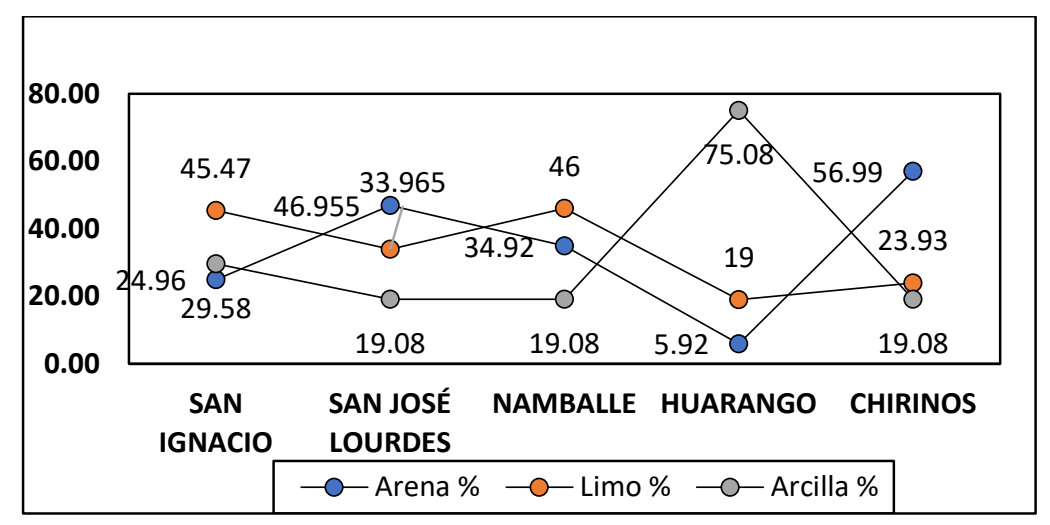

Figura 1. Parámetro textura de suelo: arena, limo, arcilla en cinco zonas cafetaleras

\section{Potencial de Hidrógeno (pH).}

En la Figura 2 se aprecia que para la variable $\mathrm{pH}$, se obtuvo un promedio de 5.16 con una desviación estándar de 0.89 de acidez, lo que representa suelos del tipo fuertemente ácido y, un coeficiente de variación de $17.2 \%$, que representa una variación media - baja.

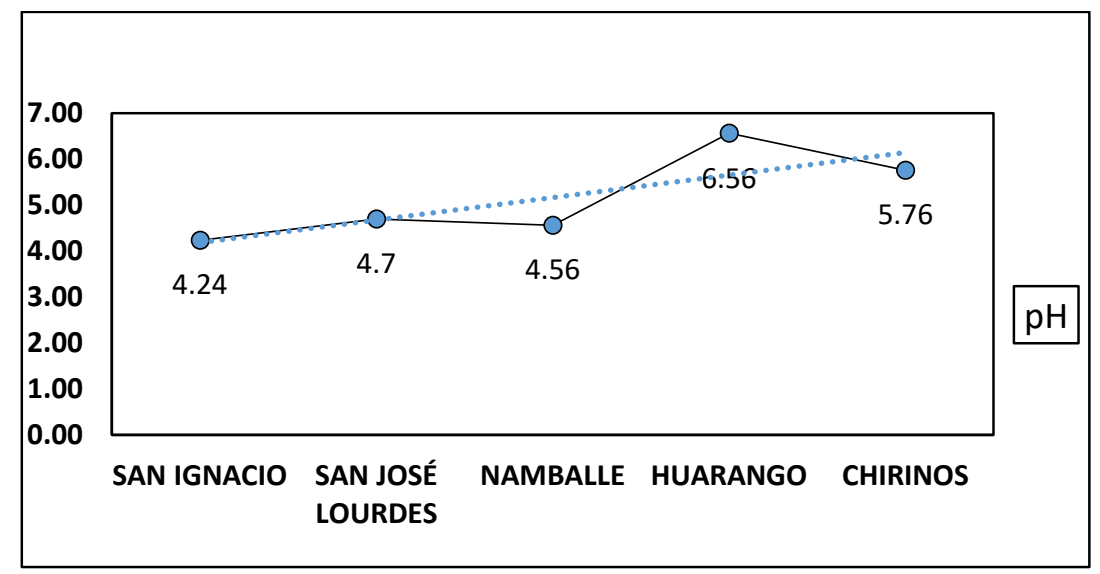

Figura 2. pH del suelo en cinco zonas cafetaleras

\section{Materia orgánica (MO).}

En la Figura 3 se puede comprobar que los suelos de la cuenca presentan una media de $4.40 \%$, con una desviación estándar de 1.43, y un coeficiente de variación de $32.49 \%$, lo que nos indica la presencia de suelos con alto contenido de materia orgánica. 
Análisis comparativos de suelos de cinco distritos de la cuenca Chinchipe

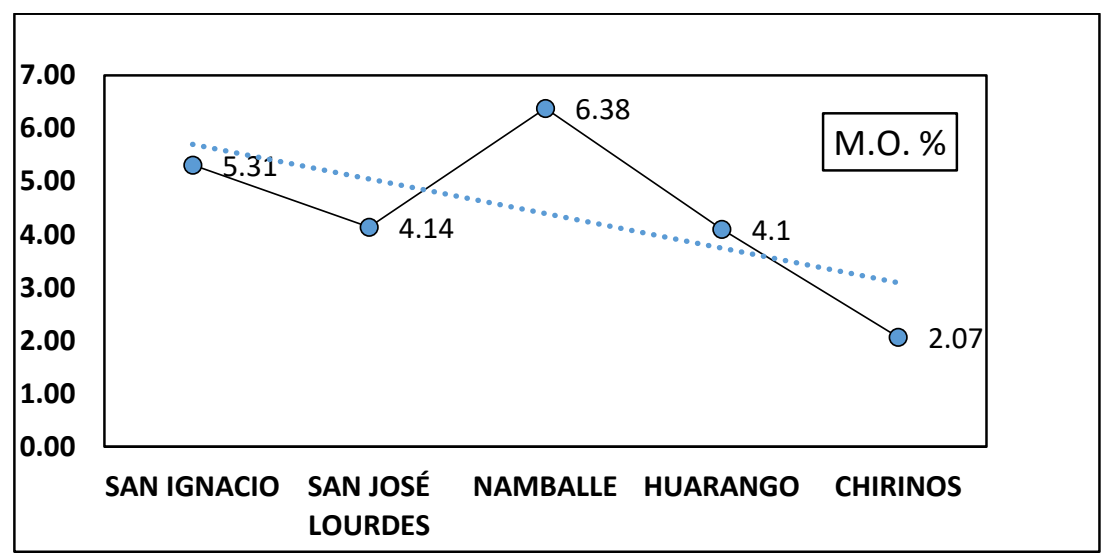

Figura 3. Contenido de materia orgánica en cinco zonas cafetaleras

\section{Porcentaje de acidez intercambiable}

En la Figura 4 se muestra que los valores medios alcanzaron $26.43 \%$, con una desviación estándar de 31.48, y un coeficiente de variación de $119.1 \%$, esto indica que se trata de suelos sin problemas de aluminio.

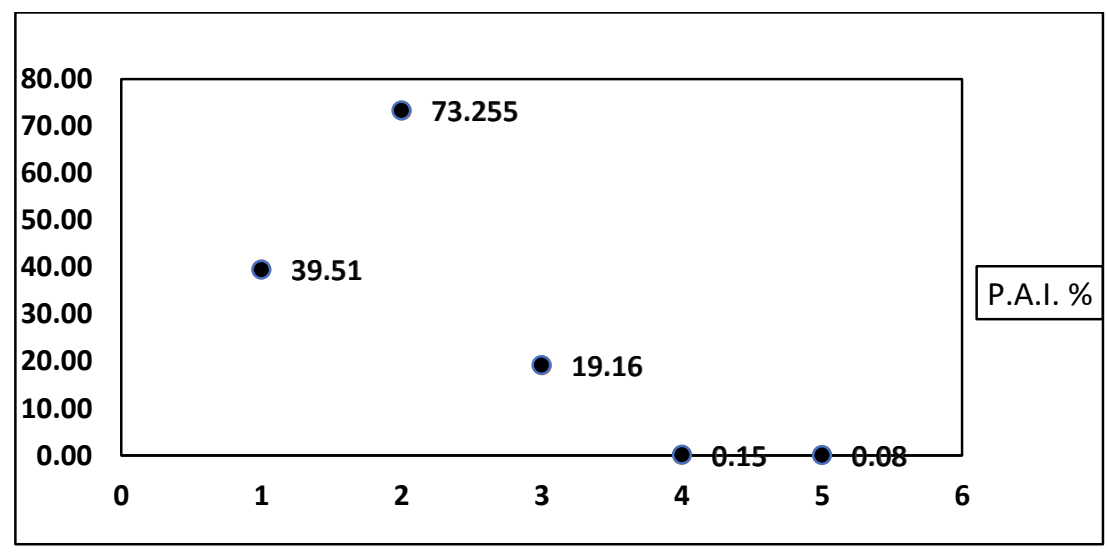

Figura 4. Porcentaje de acidez intercambiable en cinco zonas de café

\section{Capacidad de intercambio catiónico efectivo (CICE).}

En la Figura 5 se muestra la potencialidad de la fertilidad del suelo. El estudio de la cuenca, permite concluir, que, los valores medios correspondieron a $16.46 \mathrm{mEq} / 100 \mathrm{~g}$ con una desviación estándar de 15.93 y un coeficiente de variación de $96.79 \%$. 


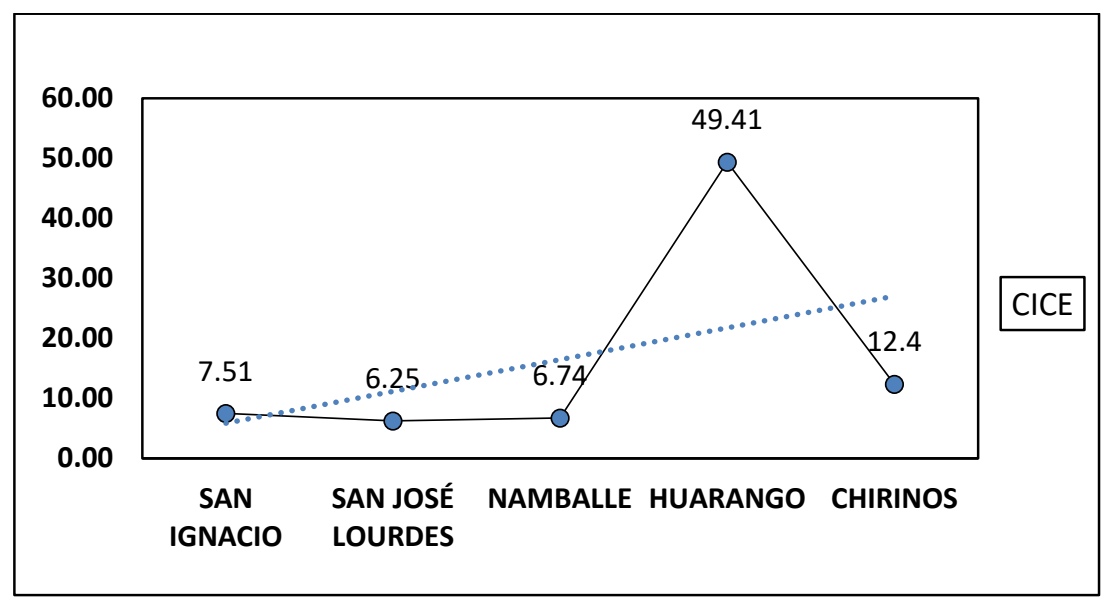

Figura 5. Capacidad de Intercambio Catiónico en cinco zonas de café

\section{DISCUSIÓN}

La cuenca del Chinchipe en esta parte de la provincia de San Ignacio, presenta tres pisos altitudinales bien definidos: la cuenca alta, desde los 2000 m.s.n.m. a los 3800 m.s.n.m.; cuenca media, desde los 1200 m.s.n.m. a los 2000 m.s.n.m.; y la cuenca baja, desde los 500 m.s.n.m. hasta los 1200 m.s.n.m. La caficultura de especialidad se ubica dentro de la cuenca media, puesto, que presenta las características agroecológicas ideales para el café de alta calidad organoléptica.

En el parámetro textura el estudio nos revela que los suelos de los cinco distritos intervenidos, presentan en promedio condiciones apropiadas para el café donde predomina el suelo del tipo Franco. Lo anterior coincide con la clasificación de texturas de suelo de la FAO en 1990. (IPGRI, 2006) determina que los suelos francos son los más apropiados para el cultivo de café. (Arcos et al., 2007), manifiesta que los suelos de la cuenca del Chinchipe, presentan buena textura, predominando los francos (arenoso, arcilloso, limoso), buena porosidad, buena profundidad efectiva $(30-40 \mathrm{~cm})$, permitiendo un normal desarrollo de la raíz, buen drenaje.

En el parámetro $\mathrm{pH}$, el estudio nos revela que los suelos, presentan rangos de $\mathrm{pH}$ ideales para la producción sostenible de café, que van desde 4.5 a 6. (Arcos et al., 2007), contribuye en su informe final que los suelos en el ámbito de la cuenca del Chinchipe, son en su mayoría inceptisoles, de $\mathrm{pH}$ ácido (4.3 - 5.0) y que manejados técnicamente son productivos. 
Para el parámetro materia orgánica, los resultados obtenidos indican que los suelos en estudio tienen un alto contenido de materia orgánica (mayor a $4 \%$ ). Es conocido que el rango ideal de materia orgánica para el cultivo de café deberá ser mayor a 4\%. (Rimache, 2008), considera que el rango medio para cafés es de 2.1 a $4 \%$, y alto de 4.1 a más.

En el parámetro acidez intercambiable, los resultados obtenidos indican que los suelos de los distritos en estudio, presentan una media del grado de dispersión con respecto al valor promedio o media $(26.43 \%)$, este diagnóstico nos permite concluir que no existe problemas de toxicidad por el elemento aluminio. La acidez intercambiable se refiere al hidrógeno y aluminio intercambiables $\left(\mathrm{H}^{+}, \mathrm{Al}^{3+}\right)$ retenidos en los coloides del suelo por fuerzas electrostáticas (Espinoza et al., 2000). Se conoce que, el rango máximo para café debe ser menor a $30 \%$.

Para el parámetro Capacidad de Intercambio Catiónico Efectivo (C.I.C.E.), el estudio revela que, se trata de suelos con alta fertilidad, esto se explica, porque, el valor medio encontrado corresponde a $16.46 \mathrm{mEq} / 100 \mathrm{~g}$ y la literatura indica que, el rango ideal para la producción sostenible debe ser mayor a $12 \mathrm{mEq} / 100$ g. Sin embargo, Namballe y San José de Lourdes presentaron los valores más bajos $(6.74 \mathrm{mEq} / 100 \mathrm{~g}$ y $6.25 \mathrm{mEq} / 100 \mathrm{~g}$ respectivamente), poniendo en evidencia que, en la práctica, los rendimientos de café por hectárea en estos distritos son bajos, por lo tanto, la fertilización adecuada en base a análisis de suelo, es una alternativa para mejorar la productividad.

Finalmente, es importante añadir como conclusión que, para complementar un análisis más detallado de los suelos en estudio, se debería investigar, el contenido de macroelementos como el nitrógeno, fósforo, potasio (NPK) y microelementos como boro, zinc (B, Zn).

\section{CONCLUSIONES}

Según los parámetros evaluados del análisis de suelos, se establece que los suelos de los distritos que forman parte de la cuenca del Chinchipe, presentan propiedades físicas apropiadas para la producción sostenibles de cafés de especialidad, la clase textural corresponde al tipo FRANCO, es decir, suelos ideales para el cultivo.

Los suelos presentan, asimismo, propiedades químicas ideales y que están dentro de los rangos establecidos para el cultivo del café. El primer parámetro químico evaluado fue el $\mathrm{pH}$, donde se obtuvo un promedio de 5.16, indicando que son suelos fuertemente ácidos, 
y se puede recomendar fertilizar con fertilizantes y enmiendas que no ocasiones acidez como la roca fosfórica, materia orgánica.

El segundo parámetro químico evaluado fue la materia orgánica que presentó un promedio de $4.40 \%$, concluyendo que se trata de suelos con alto contenido de materia orgánica. El tercer parámetro químico fue, el porcentaje de acidez intercambiable (PAI) mostrando un valor medio de $26.43 \%$, (rango ideal para café es < 30\%), esto indica que se trata de suelos sin problemas por toxicidad de aluminio. El cuarto parámetro químico evaluado fue la Capacidad de Intercambio Catiónico Efectivo (CICE) el mismo que tuvo una media de $16.46 \mathrm{mEq} / 100 \mathrm{~g}$, indicando que corresponden a suelos de alta fertilidad. Por lo tanto, el estudio realizado nos permite concluir que se trata de suelos apropiados para el cultivo del café de especialidad: a) café gourmet, b) café orgánico, c) cafés sostenibles d) micro lotes.

\section{AGRADECIMIENTOS:}

Al Economista Reiles Jaime Zapata Ramos, manager del programa familia de la empresa Comercio \& CIA. S.A.

A Julio Castro Lazo, director del Laboratorio del Instituto Rural Valle Grande Laboratorio de Química Agrícola

\section{REFERENCIAS BIBLIOGRÁFICAS}

ANACAFE (2006). Estudio Comparativo de costos de fertilización, con y sin análisis de suelos. Asociación Nacional del Café.

Arcos, M.; Karst; S. (2007). "Sistematización de Experiencias Agroforestales y Silvopastoriles en el Ámbito de PRO - SNTN". Programa de Apoyo al Desarrollo Sostenible de la Zona de Influencia del Santuario Nacional Tabaconas Namballe, en la Provincia de San Ignacio. Informe Final. 101 p.

Ayala, E.; Bernabé, E.; Montoya, M.; Pinedo; J. (2016). Planeamiento Estratégico del Café en el Perú. Tesis para obtener el grado de Magíster en Administración Estratégica de Empresas-Pontificia Universidad Católica del Perú, Lima-Perú. $162 \mathrm{p}$.

Cámara Peruana del Café y Cacao (2016). Actual Distribución de las Zonas Cafetaleras del Perú. recuperado de http://www.camcafeperu.com.pe/index.php/estadisticas 
Eliot, J.; Coello, J.; Alcalde, M. (2009). Los Bosques de la Cuenca Transfronteriza del Río Mayo-Chinchipe (Perú - Ecuador). Lima: Soluciones Prácticas-ITDG. 150 p.: il.

Espinoza, J.; Molina, E. (1999). Acidez y Encalado de los Suelos. 1ra edición. International Plant Nutrition Institute, Quito-Ecuador, Centro de Investigaciones Agronómicas, San José-Costa Rica. 46 p.

Jezeer. R.; Verweij. P.A. (2015). Café en Sistemas Agroforestales - doble dividendo para la biodiversidad y los pequeños agricultores en Perú. Hivos, The Hague, Holanda. $60 \mathrm{p}$.

JNC (2016). Cafetalero, La Nueva Generación del Café Peruano, Junta Nacional del Café. Revista informativa. $40 \mathrm{p}$.

JNC (s.f.). Toma de Muestra de Suelo para su Análisis. Proyecto: Modelo de Desarrollo Sostenible para el Café Peruano. Revista electrónica. 6 p.

Lllerena, C.; Cruz, Z.; Peña, M.; Martínez, K.; Ocaña, J. (2010). Gestión Ambiental de un Ecosistema Frágil. Los Bosques Nublados de San Ignacio, Cajamarca, Cuenca del Río Chinchipe. Lima: Soluciones Prácticas-ITDG. 134 p.: il.

Ministerio de Agricultura (2003). Caracterización de la Zonas Cafetaleras en el Perú. Programa para el Desarrollo de la Amazonía-PROAMAZONÍA. Informe final. Lima - Perú. 136 p.

Ortega, S. (2006). Diagnóstico de Línea de Base de Participación de Gobiernos Locales en Promoción del Uso Sostenible de Recursos Forestales. Proyecto Bosques del Chinchipe. Conservación Internacional Perú. Soluciones Prácticas-ITDG. Documento de trabajo. $71 \mathrm{p}$.

Rimache, M. (2008). Cultivo del Café. 1ra edición. Editorial Macro EIRL, pp. 56:75.

SOLUCIONES PRÁCTICAS (2016). Cobertura de Árboles para el Cambio Climático. Tecnología 1. Guía para la producción sostenible de la caficultura en la selva alta peruana. Ficha técnica. 8 p.

Vílchez, N.; Zapata, R. (2017). El Café en la Cuenca del Chinchipe. Programa Familia. Comercio \& Cía. S.A. Jaén - Perú. 271 p. 